\title{
Ischemic Stroke Presenting as the First Symptom in a Setting of Paroxysmal Nocturnal Hemoglobinuria
}

\author{
Muhammad Junaid Ahsan ${ }^{1}$, Rizwan Ishtiaq ${ }^{2}$, Daniyal Ishtiaq ${ }^{3}$ \\ 1. Medicine, Interfaith Medical Centre 2. Internal Medicine, St. Vincent Mercy Medical Center, Toledo, \\ USA 3. Internal Medicine, Rawalpindi Medical College, Rawalpindi, PAK
}

$\square$ Corresponding author: Muhammad Junaid Ahsan, junaidahsan333@gmail.com Disclosures can be found in Additional Information at the end of the article

\section{Abstract}

Paroxysmal nocturnal hemoglobinuria is a hematological disorder characterized by hemolytic anemia, cytopenia, and thrombotic events. Venous thrombotic events are more commonly reported. An arterial thrombosis is a rare event in paroxysmal nocturnal hemoglobinuria. We present a case of a 32-year-old female who had symptoms of stroke and on workup, she was diagnosed as a case of paroxysmal nocturnal hemoglobinuria.

Categories: Internal Medicine, Neurology, Other

Keywords: ischemic stroke, paroxysmal nocturnal hemoglobinuria, neutropenia

\section{Introduction}

Paroxysmal nocturnal hemoglobinuria $(\mathrm{PNH})$ is characterized by a triad of complementmediated hemolysis, cytopenia and venous thrombosis [1]. In paroxysmal nocturnal hemoglobinuria (PNH), a mutation of X-chromosomal phosphatidylinositol Nacetylglucosaminyl transferase subunit A (PIG-A) gene disorganizes the synthesis of glycosylphosphatidylinositol (GPI)-linked surface proteins which are the membrane inhibitors of CD59 and CD55 [2]. Common sites for the development of venous thromboembolism include liver, abdomen and the skin [3]. Thrombosis of the cerebral arteries in the patients with PNH is a rare entity and only a few cases have been reported in the literature to date. Informed consent statement was obtained for this study.

\section{Case Presentation}

Received 06/04/2017

Review began 06/29/2017 Review ended 06/29/2017 Published 07/07/2017

(c) Copyright 2017

Ahsan et al. This is an open access article distributed under the terms of the Creative Commons Attribution License CC-BY 3.0., which permits unrestricted use, distribution, and reproduction in any medium, provided the original author and source are credited.
A 32-year-old normotensive, non-diabetic Asian female who was G5P3A2 presented to the emergency room with a one-day history of motor aphasia, weakness of left side of the body and two episodes of fits with an altered state of consciousness for the last 12 hours. Her vitals were normal with a blood pressure of $130 / 80 \mathrm{mmHg}$, a pulse of 80 beats per minute, afebrile and respiratory rate of $18 / \mathrm{min}$. The patient looked pale and mildly icteric. Glasgow coma scale was $5 / 15$. On physical examination, left plantar was up going and power was $2 / 5$ in left upper and lower limb. Reflexes were brisk and hypertonia was noted. A non-contrast computed tomography (CT) scan was performed and a hypodense area in the right frontal region was found which suggested an infarction. Multiple hypodense areas in the right frontoparietal region were also seen. CT scan of the patient and flair images of the brain are shown in Figure $1-2$. 


\section{Cureus}

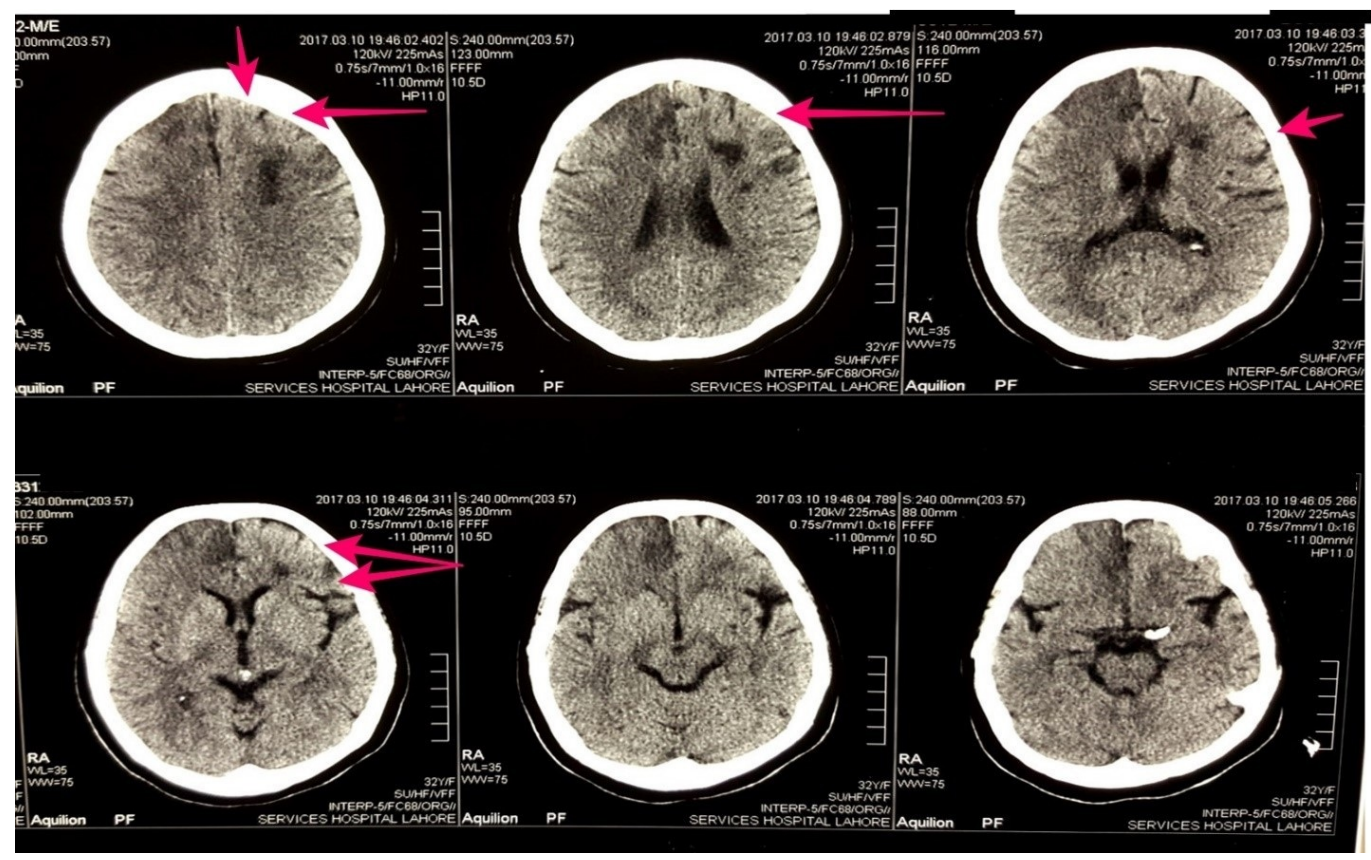

FIGURE 1: Figure showing the computed tomography scan of the patient without contrast. Red arrows show the hypodense areas in the right frontoparietal region

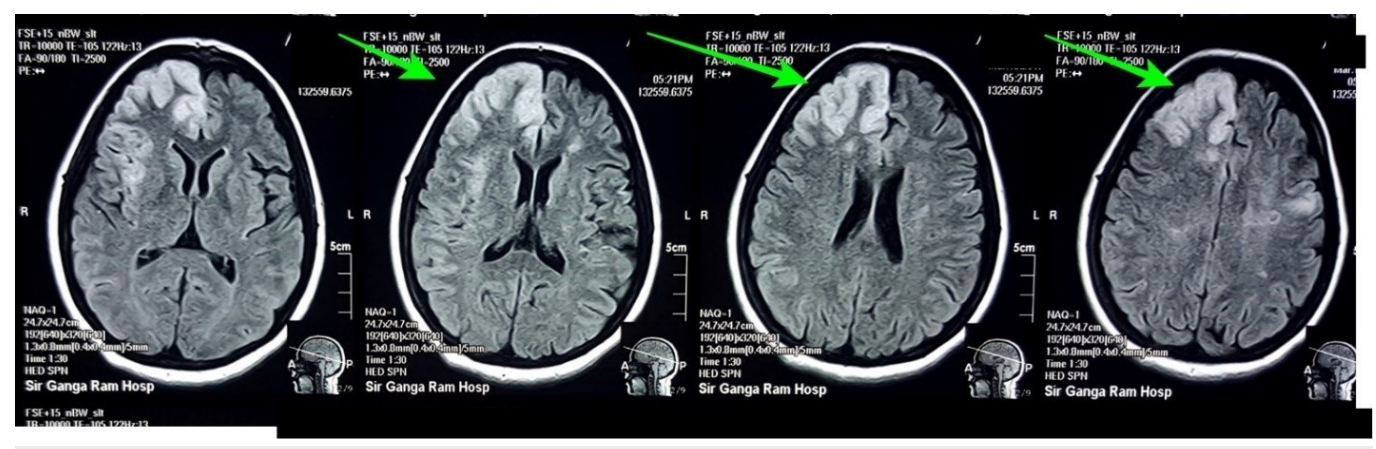

FIGURE 2: Figure showing the flair Images of the brain of the patient. Green arrows show infarct involving the right frontoparietal region of the brain

Echocardiography showed 60\% ejection fraction and carotid Doppler was normal. The patient was started on aspirin, atorvastatin, and warfarin. Foley catheter was passed and aspiration precautions and bed sore measures were instructed. On laboratory investigations, the patient had anemia with a hemoglobin of $7.5 \mathrm{~g} / \mathrm{dL}$ with the mean corpuscular volume of $106 \mathrm{fL}$, total leukocyte count of 3.2 and platelets was $241000 / \mathrm{mm} 3$. Lipid profile, liver function tests, and renal function tests were normal except a rise in indirect bilirubin levels. the patient's' stroke started to improve on the second day with patient becoming conscious on the third day of admission. The power of the left upper and lower limb returned to normal by the seventh day. During this time, the urine bag was found to have cola colored urine. Hemoglobin concentration dropped to $5.6 \mathrm{~g} / \mathrm{dL}$ even after getting packed cell volume transfusions in that week. Based on raised bilirubin levels, blood in urine, pallor, and jaundice, the patient was 


\section{Cureus}

thought to be having some sort of hemolytic anemia. There was no history of a sore throat, arthralgia, photosensitivity, alopecia and mouth ulceration ruling out systemic lupus erythematosus. Coombs test was performed which came out negative, lactate dehydrogenase (LDH) was $768 \mathrm{U} / \mathrm{L}$, haptoglobin $12 \mathrm{mg} / \mathrm{dL}$, reticulocyte count was 28 and coagulation profile was normal. The patient had a history of first two trimester abortions and further testing was performed. The patient tested negative for antinuclear antibodies (ANA), Anti-double-stranded deoxyribonucleic acid (Anti-dsDNA), Beta-2 microglobulin, and venereal disease research laboratory test (VDRL). Ham's test was performed and the result came out positive. Flow cytometry was performed and below the normal values of CD55 and CD59 were recorded and the patient was diagnosed as a case of PNH with arterial thrombosis.

She was started on corticosteroids and tablet azathioprine $50 \mathrm{mg}$ on the 10 th day but on the 14 th day, she started to get a high-grade fever with spikes ranging from 100-103F. Fever was continuous and not responsive to Panadol. Blood cultures came out negative. Chest X-ray, urine analysis, ultrasound abdomen, and pelvis were normal. On repeat complete blood count on the 14th day, the patient was found to have neutropenia along with anemia. Leukocyte count was 1.1 with an absolute neutrophilic count of $373 / \mathrm{mm} 3$. The patient was started on vancomycin B.D and piperacillin/tazobactam on eight hourly basis. By the 18th day, patient's fever started to settle down and totally afebrile by the 20th day. Leukocyte count started to rise again on the 17th day and was 1.5 on the 18th day. It followed an increasing trend and became 2.6 on the 22nd day. Trends of neutrophils rise and fall are shown in Figure 3. Antibiotics were continued for 10 days and the patient was discharged on the 25th day after her leukocyte count became 3.1. The patient was discharged on azathioprine 50mg O.D, tablet folic acid, vitamin $\mathrm{B} 12$, and warfarin.

\section{Trend in Total Leukocyte Count following admission in the hospital}

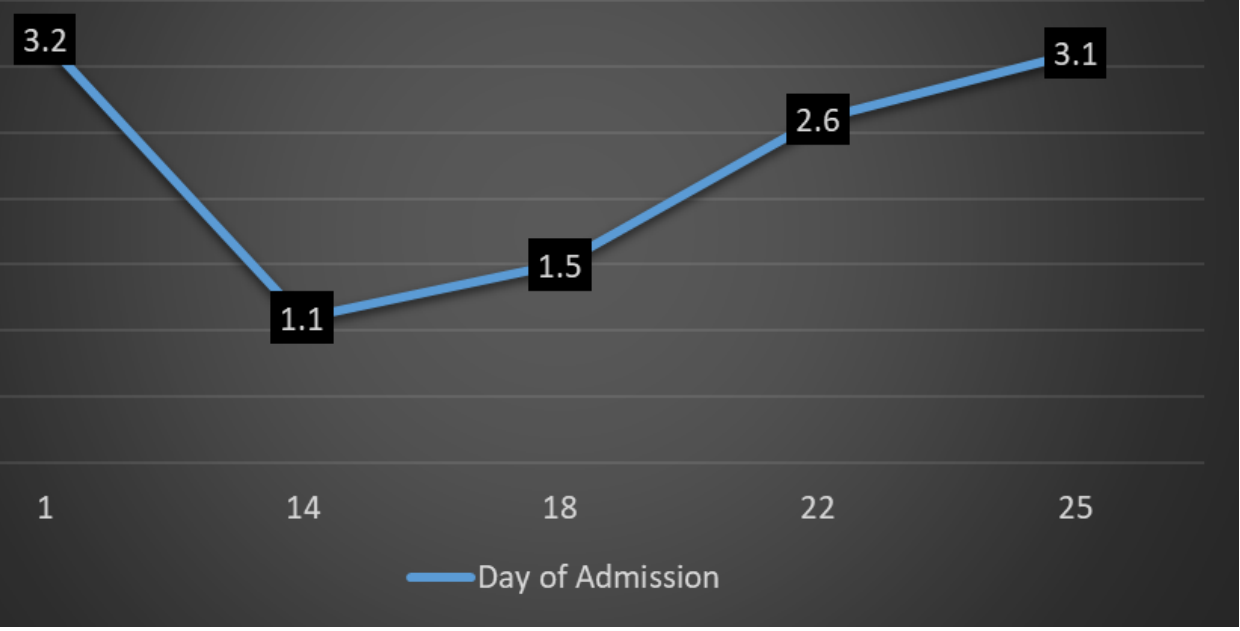

\section{FIGURE 3: Figure showing the trends of neutrophils rise and fall of the patient}

\section{Discussion}

Several mechanisms have been proposed in the literature for thrombosis in patients with PNH. Hemolyzed red blood cells releasing thromboplastic material increased platelet activation in PNH patients and the procoagulant effect produced in the plasma by platelet-derived microparticles constitute these mechanisms [4]. The fact that the presentation of stroke during 
anemia supports the hypothesis that vascular accidents are linked with the hemolytic process.

Brubaker, et al. studied the life span of neutrophils in PNH patients [5]. They observed that the neutrophils lysis rate increased in PNH patients in comparison to controls when these cells were exposed to acidified fresh serum plus serum containing Anti-neutrophilic antibodies. Although the rate of lysis of neutrophils was increased, the intravascular life span of labeled neutrophils as measured by 32P-diisopropylfluorophosphate technique remained normal. This hypothesizes that the cause of neutropenia in patients with PNH is related to decreased production or increased lysis and not related to their life span.

Flow cytometry of the red blood cells to reveal decreased or reduced expression of CD55 and CD59 remains the gold standard test for PNH [6]. Treatment consists of heparin or low molecular weight heparin with long-term anticoagulation provided by warfarin [3]. In patients having venous thrombosis from PNH, lifetime anticoagulation is recommended [3]. Other medications include folic acid, iron replacement therapy, blood transfusion and corticosteroids [7]. Median survival time in patients with PNH is 10-15 years with most of the deaths occurring in the sixth decade of life, and thromboembolic complications constitute directly to the $60 \%$ of the deaths occurring in $\mathrm{PNH}[1,3]$.

Recently, there has been a lot of debate on the growing evidence of eculizumab which is a humanized monoclonal antibody. This drug binds to C5 inhibiting terminal complement system and blocking the formation of membrane attack complex [8]. Survival rate at five years in patients with PNH was $67 \%$ and with the use of eculizumab, this rate has improved to $96 \%$. The rate of thrombotic events has also decreased with this medication. Previously, it was $6 \%$ per year and now it is less than 1\% per year [7]. Eculizumab is an expensive medication and due to non-availability in our center, we were not able to use this medication for our patient. In cases of failed treatment or refractory to medication, a bone marrow transplant is an alternative option with long-term success [9]. Mortality related to bone marrow transplant is $42 \%$ [10].

For future considerations, a phase 2 clinical trial of the safety and efficacy of eculizumab in hemolytic PNH patients has been completed. Results have not been reported yet. Another phase 2 clinical trial is currently recruiting patients to study the efficacy of covers in PNH patients with resistance to eculizumab.

\section{Conclusions}

Paroxysmal nocturnal hemoglobinuria (PNH) must be considered in the differential diagnosis of stroke in young children and adults with hemolysis. In previously undiagnosed patients with $\mathrm{PNH}$, ischemic stroke can be the only initial presentation. PNH testing should also be made a part of thrombophilia screening as thrombophilia serves as an unrecognized cause of unprovoked thrombosis. Anticoagulation should be started urgently taking into consideration both long-term anticoagulation and bone marrow transplant. Better results can be produced with multidisciplinary involvement especially a hematologist.

\section{Additional Information}

\section{Disclosures}

Human subjects: Consent was obtained by all participants in this study. Services Institute of Medical Sciences issued approval 112. Conflicts of interest: In compliance with the ICMJE uniform disclosure form, all authors declare the following: Payment/services info: All authors have declared that no financial support was received from any organization for the submitted work. Financial relationships: All authors have declared that they have no financial relationships at present or within the previous three years with any organizations that might 
have an interest in the submitted work. Other relationships: All authors have declared that there are no other relationships or activities that could appear to have influenced the submitted work.

\section{References}

1. Hillmen P, Lewis SM, Bessler M, et al.: Natural history of paroxysmal nocturnal hemoglobinuria. N Engl J Med. 1995, 333:1253-9. 10.1056/NEJM199511093331904

2. Bessler M, Mason PJ, Hillmen P, et al.: Somatic mutations and cellular selection in paroxysmal nocturnal haemoglobinuria. The Lancet. 1994, 343:951-3. 10.1016/S0140-6736(94)90068-X

3. Ray JG, Burows RF, Ginsberg JS, et al.: Paroxysmal nocturnal hemoglobinuria and the risk of venous thrombosis: review and recommendations for management of the pregnant and nonpregnant patient. Pathophysiol Haemost Thromb. 2000, 30:103-17. 10.1159/000022532

4. Gralnick HR, Vail M, McKeown LP, et al.: Activated platelets in paroxysmal nocturnal haemoglobinuria. Br J Haematol. 1995, 91:697-702. 10.1111/j.1365-2141.1995.tb05371.x

5. Brubaker LH, Essig LJ, Mengel: Neutrophil life span in paroxysmal nocturnal hemoglobinuria . Blood. 1977, 50:657-62.

6. Olteanu $\mathrm{H}, \mathrm{Xu} \mathrm{Y}$ : The use of flow cytometry in diagnosis of paroxysmal nocturnal hemoglobinuria. Lab Med. 2006, 37:498-502. 10.1309/NAAH5J8DV43C0H7N

7. Memon AR, Khan R, Rauf MU, et al.: Paroxysmal nocturnal hemoglobinuria presenting as cerebral venous sinus thrombosis: A case report.. Int Arch Med. 2014, 7:39.

8. Rother RP, Rollins SA, Mojcik CF, et al.: Discovery and development of the complement inhibitor eculizumab for the treatment of paroxysmal nocturnal hemoglobinuria. Nat Biotechnol. 2007, 25:1256-64. 10.1038/nbt1344

9. Raiola AM, Van Lint MT, Lamparelli T, et al.: Bone marrow transplantation for paroxysmal nocturnal hemoglobinuria. Haematologica. 2000, 85:59-62.

10. Santarone S, Bacigalupo A, Risitano AM, et al.: Hematopoietic stem cell transplantation for paroxysmal nocturnal hemoglobinuria: long-term results of a retrospective study on behalf of The Gruppo Italiano Trapianto Midollo Osseo (GITMO). Haematologica. 2010, 95:983-8. 10.3324/haematol.2009.017269 